\title{
Psicooncología
}

ISSN: 1696-7240

\section{Validación de la escala QLQ-C15 PAL, para evaluar calidad de vida en pacientes en cuidados paliativos, al español colombiano}

\author{
Marcela Andrea Erazo Muñoz ${ }^{1 *}$; Yonatan Rojas Salgado²; Olga María Vargas ${ }^{3}$; Claudia \\ Carolina Colmenares Mejía ${ }^{4}$
}

Recibido: 3 de octubre de 2020 / Aceptado: 2 de mayo de 2021

Resumen. Objetivo: Validar la escala QLQ C15 PAL, para evaluar calidad de vida en pacientes en cuidados paliativos, al español colombiano, considerando un análisis psicométrico desde la teoría clásica de los test. Método: Muestra por conveniencia de 155 pacientes oncológicos atendidos por el Programa de Cuidados Paliativos de un grupo de centros hospitalarios en las ciudades de Bogotá y Cali en Colombia. Para estimar la validez de constructo, se utilizó el análisis factorial exploratorio (AFE) con rotación varimax; posteriormente, se usaron cargas factoriales para estimar el coeficiente Omega y posteriormente el análisis de Rash reconocido Análisis de la Teoría de la respuesta al ítem (TRI). El análisis fue realizado en Winsteps 2.80. Resultados: Todas las correlaciones son significativas entre los ítems de la escala Funcionamiento Global (FG) con el puntaje total obtenido en esta escala y el puntaje total del QLQ PAL-15; la misma situación ocurre con los ítems de la escala Funcionamiento Emocional (FE) que correlaciona de modo directamente proporcional y con una magnitud alta con el puntaje total del QLQ PAL-15. En cuanto al total de la escala, existe un adecuado ajuste para los ítems ya que sus valores Infit: (IMNSQ 0,99; ZSDT: -0,3) y Outfit (OMNSQ 1,02; ZSDT-0,2) se encuentran dentro de los rangos establecidos como parámetros de ajuste. Conclusiones: La validación del EORTC QLQC15-PAL demostró propiedades psicométricas satisfactorias al aplicarse en pacientes con enfermedad oncológica avanzada en Colombia. Se plantea para estudios posteriores realizar ajuste en la redacción de los ítems 7,11 y 13 para que estos discriminen de manera precisa el rasgo que pretenden medir Palabras claves: Calidad de vida, cuidados paliativos, estudios de validación, enfermo terminal.

\section{[en ] Validation of the QLQ-C15 PAL Scale, to assess quality of life in patients in palliative care, to colombian spanish}

Abstract. Objective: Validate the QLQ C15 PAL scale, to assess the quality of life in patients in palliative care, to Colombian Spanish, based in a classical psychometric analysis. Methodology:

1 Marcela Andrea Erazo Muñoz Unidad para el alivio del dolor y cuidado paliativos, Clínica Reina Sofía. Bogotá, Colombia.

E-mail: maerazo@colsanitas.com

2 Yonatan Rojas Salgado.Facultad de psicología, Fundación Universitaria Sanitas. Bogotá, Colombia.

E-mail: yfrojas@unisanitas.edu.co

3 Olga María Vargas. Fundación Cardioinfantil Colombia. Universidad de los Andes. Bogotá, Colombia.

E-mail: olgamariavargas@live.com

4 Claudia Carolina Colmenares Mejía. Unidad de investigaciones, Fundación Universitaria Sanitas. Bogotá, Colombia.

E-mail: cccolmenaresm@unisanitas.edu.co

* Correspondencia: Marcela Erazo Muñoz. Unidad para el alivio del dolor y cuidado paliativos, Clínica Reina Sofía. Bogotá, Colombia. E-mail: maerazo@colsanitas.com

Fuente de financiación: Clínica Colsanitas. Fundación Universitaria Sanitas. 
Convenience sample of 155 cancer patients attended by the Palliative Care Program of a group of hospitals in the cities of Bogota and Cali in Colombia. To estimate construct validity, exploratory factor analysis (AFE) with varimax rotation was used; Factorial loads were subsequently used to estimate the Omega coefficient and subsequently Rash's recognized Analysis of Item Response Theory (TRI). The analysis was performed on Win steps 2.80. Results: All correlations are significant between the items on the Global Operation (FG) scale with the total score obtained on this scale and the total score of the QLQ PAL-15; the same situation occurs with items on the Emotional Functioning (FE) scale that correlates directly proportionally and with a high magnitude with the total score of QLQ PAL-15. As for the total scale, there is an appropriate adjustment for the items since their Infit values: (IMNSQ 0.99, ZSDT: -0.3) and Outfit (OMNSQ 1.02, ZSDT-0.2) are within the ranges set as adjustment parameters. Conclusion: The validation of the EORTC QLQ-C15-PAL demonstrated satisfactory psychometric properties in patients with advanced cancer disease in Colombia. It is proposed for subsequent studies to adjust the wording of items 7,11 and 13 so that they discriminate in a precise way the feature that they intend to measure.

Keywords: Quality of life, palliative care, measurement instruments, end of life care.

Sumario: 1. Introducción 2. Método 3. Análisis 4. Resultados 5. Discusión. 6. Conclusión. 7. Referencias bibliográficas

Como citar: Erazo Muñoz MA., Rojas Salgado Y, Vargas OM, Colmenares Mejía CC. Validación de la escala QLQ-C15 PAL, para evaluar calidad de vida en pacientes en cuidados paliativos, al español colombiano. Psicooncología 2021; 18: 359-369. https://dx.doi.org/10.5209/psic.77758

\section{Introducción}

El cáncer es una de las causas principales de muerte alrededor del mundo. En 2018, los datos mundiales de GLOBOCAN sobre el cáncer sugieren que la carga mundial ha aumentado llegando a 18,1 millones de casos nuevos y 9,6 millones de muertes. En Colombia, en el año 2018, se presentaron 101.000 casos nuevos, la tasa de incidencia por edad es de 180 casos nuevos por cada 100.000 personas y 46 mil personas fallecieron por esta causa ${ }^{(1)}$.

Como parte del curso propio de la enfermedad se evidencia el deterioro progresivo en la perdida de funcionalidad, presencia de síntomas físicos, emocionales y espirituales que interfieren con la calidad de vida necesitando intervención interdisciplinar para esta población, por lo que la OMS ha considerado la inclusión de los Cuidados Paliativos como el "Planteamiento que mejora la calidad de vida de los pacientes (adultos y niños) y sus allegados cuando afrontan problemas inherentes a una enfermedad potencialmente mortal. Previenen y alivian el sufrimiento a través de la identificación temprana, la evaluación y el tratamiento correctos del dolor y otros problemas, sean estos de orden físico, psicosocial o espiritual ${ }^{(2) "}$.

De acuerdo con lo anterior se ha hecho necesario contar con instrumentos de medición validados, adaptados y objetivos para evidenciar el impacto tanto de la enfermedad como del tratamiento en la calidad de vida de los pacientes.

La Organización Europea para la investigación y tratamiento del cáncer (EORTC por sus siglas en inglés) ha desarrollado diferentes instrumentos para evaluar la calidad de vida; en particular para pacientes en cuidados paliativos sugiere el uso del EORTC QLQ C15 PAL que es una versión corta del QLQ-C30 ${ }^{(3)}$. E1 QLQ C15 PAL se diseñó para evaluar la calidad de vida en pacientes con patología oncológica en 
estadio avanzado, quienes presenten síntomas asociados a la enfermedad y tengan una expectativa de vida de pocos meses.

Es sabido que la medición de constructos no solo considera la estructura interna del instrumento, sino que los ítems deben ser sensibles a la cultura y estar ajustados semántica y semióticamente al contexto al que pertenecen los sujetos a quienes se dirige la prueba; es por esto por lo que las propiedades psicométricas de un test deben siempre calcularse para cada población en la que se usa el test incluso cuando proviene del mismo idioma ${ }^{(4)}$.

El objetivo del presente estudio es validar la escala QLQ C15 PAL, para evaluar calidad de vida en pacientes en cuidados paliativos, al español colombiano, mediante un análisis psicométrico desde la teoría clásica de los test ${ }^{(5)}$ considerando para ello los supuestos de linealidad en la medida ${ }^{(6)}$, así como los de la teoría de la respuesta al ítem ${ }^{(7)}$, para establecer el grado de ajuste individual de cada uno de los ítems ${ }^{(8)}$, de modo tal que el documento reúna suficiente evidencia para garantizar la precisión en la medida de la calidad de vida relacionada con la salud.

\section{Método}

\section{Diseño}

Este es un estudio observacional de corte transversal y de temporalidad prospectiva. De acuerdo con el tipo de análisis de datos que se realizó para el estudio de confiabilidad y validez, la conformación de la muestra se hizo por oportunidad hasta completar 155 pacientes oncológicos atendidos por un programa de cuidados paliativos en Colombia, garantizando los principios de homocedasticidad y normalidad, que requieren una muestra que tenga una distribución normal ${ }^{(9)}$.

\section{Muestra}

Para la selección de los participantes, se definieron los siguientes criterios de inclusión a) pertenecer al programa de cuidados paliativos de una de las instituciones participantes, b) tener más de 18 años, c) haber aceptado participar mediante la firma de consentimiento informado, d) haber demostrado conocer y comprender las instrucciones de la tarea y e) haber completado el total de la actividad de evaluación. Se excluyeron los sujetos que tenían un déficit en lecto-escritura o alteración en procesos de comprensión y seguimiento de instrucciones.

\section{Instrumento de Evaluación.}

El instrumento EORTC QLQ C15 PAL ${ }^{(10)}$ fue desarrollado como versión corta de 15 ítems para los pacientes con enfermedad oncológica avanzada en fin de vida, de la versión de 30 ítems (EORTC-QLQ-C30) que fue desarrollado por la European Organization for Research and Treatment of Cancer (EORTC) $)^{(3)}$ para evaluar la calidad de vida (QLQ), a través de un cuestionario que incorpora temas sobre la salud física, emocional y social para los pacientes con cáncer. Esta escala fue previamente adaptada transculturalmente en una muestra de pacientes con cáncer en Colombia ${ }^{(11)}$. E1 QLQ C15 PAL, está compuesto por 15 ítems, organizados en escala sintomática 
que corresponde a los Ítems 4 al 12: evalúa dolor, disnea, insomnio, pérdida de apetito y estreñimiento; la escala de funcionamiento global incluye los ítems 1,2 y 3 , la escala de funcionamiento emocional los ítems 13 y 14 y la evaluación de calidad de vida global que corresponde al ítem $15^{(12)}$. Para obtener el puntaje se realiza una transformación lineal del valor dado por el paciente a una puntuación de 0 a 100 , siendo 100 la mejor salud global o mejor calidad de vida.

\section{Procedimiento.}

La recolección de datos se realizó entre febrero y septiembre de 2017 en pacientes oncológicos atendidos por un programa de cuidados paliativos en Colombia. Se incluyeron dos clínicas (atención hospitalaria y ambulatoria) de la ciudad de Bogotá, una clínica de la ciudad de Cali y el programa de hospitalización domiciliaria de estas ciudades. Cada una de estas sedes cuenta con un equipo interdisciplinario integrado por médicos generales, médicos especialistas en cuidados paliativos, enfermeras, psicólogas, trabajadoras sociales y consejeros espirituales.

A partir de la información contenida en la historia clínica de cada paciente, se recolectó información relacionada con características demográficas, tipo de diagnóstico oncológico, Índice de Karnofsky ${ }^{(13,14)}$. Además, se diligenció por parte de los pacientes en su respectivo lugar de atención (consulta externa, hospitalización o domicilio) el instrumento QLQ C15 PAL, en la versión aportada por el EORT que no se encuentra validada en Colombia.

\section{Análisis}

Las variables cualitativas se describen como frecuencias absolutas y relativas, mientras que la edad se presenta a través de medianas y Rangos Intercuartílicos (RIQ) dado su comportamiento estadístico (verificado por la prueba de Shapiro Wilks). El índice de Karnofksy se reporta como promedio y desviación estándar; se estimó una correlación de Spearman, considerando el tipo de escala de los puntajes ${ }^{(5)}$ entre los ítems, su escala y puntaje total para probar el paralelismo entre cada ítem y el puntaje total ${ }^{(7)}$.

Para estimar la validez de constructo, se consideró mediante un análisis factorial exploratorio (AFE) con rotación varimax y un método de extracción por componentes principales ya que se pudo evidenciar que la distribución de los datos no se encontraba tipificada ${ }^{(6)}$; posteriormente se usaron estas cargas factoriales para estimar el coeficiente Omega, un coeficiente que se ha reportado como efectivo para la estimación de la consistencia interna ${ }^{(15)}$ y que es una alternativa para el clásico Alpha de Cronbach ${ }^{(16)}$.

Posteriormente se practicó un análisis de Rash reconocido Análisis de la Teoría de la respuesta al ítem (TRI) considerando la estimación de los valores de ajuste al modelo (Infit - Outfit) ${ }^{(8)}$, del mismo modo se consideraron las medidas de correlación biserial puntual, como evidencia de discriminación del cada uno de los ítems del test; posteriormente se optó por la visualización de la calibración de los ítems mediante el mapa de ítems y personas ${ }^{(17)}$, el cual es una forma de establecer el ajuste que existe entre los grados de sensibilidad de los ítems y los grados de calidad de vida de los pacientes evaluados. El análisis fue realizado en Winsteps 2.80 diseñado para el ejecutar el Análisis de Rash ${ }^{(18)}$. 
El proyecto se presentó al comité de investigaciones de la facultad de medicina y psicología para su aprobación inicial, se aprobó en la comisión de investigación y posteriormente se obtuvo el aval del comité de ética institucional.

\section{Resultados}

Se incluyeron 155 pacientes pertenecientes al programa de cuidados paliativos de las instituciones participantes. Las características de los pacientes se presentan en la tabla 1. En promedio el Índice de Karnofsky indica que la mayoría de los pacientes presentaban funcionalidad limitada, necesitando ayuda para la realización de actividades de la vida cotidiana y atención frecuente del equipo de salud. El 57\% de los pacientes estaban siendo atendidos en unidades de cuidados paliativos de hospitales generales y $43 \%$ en hospitalización domiciliaria.

Tabla1. Características de los sujetos incluidos

\begin{tabular}{lc}
\hline \multicolumn{1}{c}{ Variable } & $\begin{array}{c}\mathbf{N}=\mathbf{1 5 5} \\
\mathbf{n}(\mathbf{\%})\end{array}$ \\
\hline Edad* & $71(62-80)$ \\
\hline Género & \\
Femenino & $97(62,6)$ \\
Masculino & $58(37,4)$ \\
\hline Diagnóstico & \\
Colon o recto & $21(13,5)$ \\
Pulmón & $18(11,6)$ \\
Próstata & $15(9,7)$ \\
Hígado o vías biliares & $14(9,0)$ \\
Ovario & $13(8,4)$ \\
Estómago o esófago & $13(8,4)$ \\
Mama & $10(6,4)$ \\
SNC, columna o médula espinal & $10(6,4)$ \\
Enfermedades hematológicas & $8(5,2)$ \\
Vía urinaria & $7(4,5)$ \\
Páncreas & $7(4,5)$ \\
Otros & $19(12,3)$ \\
\hline Índice de Karnofsky** & $49,2(15,7)$ \\
Femenino & $51,5(14,5)$ \\
Masculino & $45,5(17,1)$ \\
\hline
\end{tabular}

*Mediana (RIQ). **Promedio (DE)

Los hallazgos sobre la validez de constructo corresponden a la correlación de los ítems con el puntaje de cada escala, como se muestra en la tabla 2; todas las correlaciones son significativas entre los ítems de la escala Funcionamiento Global (FG1; FG2 y FG3) con el puntaje total obtenido en esta escala y el puntaje total del QLQ PAL-15; la misma situación ocurre con los ítems de la escala Funcionamiento 
Emocional (FE 13 y FE 14) que correlaciona de modo directamente proporcional y con una magnitud alta con el puntaje total del QLQ PAL-15 y con el puntaje total obtenido en la escala FE. Estos hallazgos muestran que existe consistencia en la medida de los ítems de estas dos escalas

Los análisis de correlación indican que la escala de síntomas (ES) contiene algunos ítems que tienen una baja correlación con el puntaje total de la dimensión (ES) como con el puntaje total del QLQ PAL-15, específicamente el ítem ES4 no aporta a la consistencia de esta dimensión del cuestionario y contribuye al error de medición del QLQ PAL-15. En la tabla 2 se aprecian correlaciones significativas de baja magnitud $(<0,40)$ entre ítems que pertenecen a la Escala Sintomática y otras escalas del QLQ PAL-15, lo que constituye evidencia de unidimensionalidad de los ítems del test con respecto al constructo que pretende medir e independencia local de los ítems del cuestionario que muestran que si bien existe asociación general de los ítems del test, puesto que miden el mismo constructo (calidad de vida), poseen su propia identidad de medición.

Finalmente, se puede apreciar de manera preliminar la independencia del ítem 15 (CVG); esto puede explicarse por el escalamiento del ítem y el intervalo de puntuación y considerando esta situación, se procedió a la siguiente fase de análisis omitiendo este ítem, atendiendo a las recomendaciones de la literatura psicométrica reciente sobre la adaptación transcultural de cuestionarios y que recomiendan reducir las fuentes de variabilidad de un test haciendo análisis dimensional del cuestionario, agrupando solo los ítems de cada dimensión; en este caso, al considerar el ítem 15 como una escala independiente, se omitió en los siguientes procedimientos de análisis ya que no presenta la comunalidad necesaria para que sea procesado con una técnica factorial.

Tabla 2. Coeficientes de correlación de Spearman Ítem-Escala

\begin{tabular}{|c|c|c|c|c|c|c|c|c|c|c|c|c|c|c|}
\hline & FG1 & FG2 & FG 3 & ES4 & ES5 & ES6 & ES7 & ES8 & ES9 & ES10 & ES11 & ES12 & FE13 & FE14 \\
\hline $\begin{array}{l}\text { Funcionamiento } \\
\text { Global (FG) }\end{array}$ &, $928 * *$ &, $936 * *$ &, $940 * *$ &, $369 * *$ &, $388 * *$ &, $357 * *$ &, $689 * *$ &, $558 * *$ &, $329 * *$ &, $380 * *$ & , $698 * *$ &, $472 * *$ & , $173 *$ &, $408^{* *}$ \\
\hline $\begin{array}{l}\text { Escala de } \\
\text { Síntomas (ES) }\end{array}$ &, $631 * *$ &, $656^{*}$ &, $611 * *$ &, $540^{*}$ & ,687* &, $601 *$ &, $808 * *$ &, $743 * *$ &, $642 * *$ & , $618 *$ &, $827 * *$ &, $778 * *$ & , $367 *$ &, $620 * *$ \\
\hline $\begin{array}{l}\text { Funcionamiento } \\
\text { Emocional (FE) }\end{array}$ &, $259 * *$ &, $327^{*}$ &, $337 * *$ &, $305^{*}$ &, $447 * *$ &, $610 * *$ &, $469 *$ & , $352 *$ & , $216^{*}$ &, $233 * *$ &, $452 * *$ & ,469*, & ;, $886 * *$ &, $890 * *$ \\
\hline $\begin{array}{l}\text { Pregunta } 15 \\
(\mathrm{CVG})\end{array}$ &,$- 397 * *$ &,$- 377 * *$ &,$- 287 * *$ &,$- 232 * *$ &,$- 320 * *$ &,$- 261 * *$ &,$- 298 * *$ &,$- 319 * *$ &,$- 234 *$ &,- 069 &,$- 251^{* *}$ &,$- 251^{*}$ &,$- 165^{*}$ &,$- 327^{* *}$ \\
\hline Puntaje Total &, $738 * *$ &, $722 * *$ &, $753^{* *}$ &, $518^{*}$ &, $624 *$ & , $599 *$ &, $832 * *$ &, $717 * *$ & ,608 & ,590 &, $851^{* *}$ &, $737^{* *}$ &, $437 *$ &, $674^{* *}$ \\
\hline
\end{tabular}

** La correlación es significativa en el nivel 0,01(Bilateral)

* La correlación es significativa en el nivel 0,05 (Bilateral)

Continuando la verificación de la validez de constructo del cuestionario se procedió a confirmar los supuestos del análisis factorial exploratorio (AFE); se demostró Inter correlación entre los ítems con un KMO alto $(0,885)$ y la prueba de esfericidad demuestra correlaciones significativamente diferentes de $0(=1430,88 \mathrm{p}<0,001)$, superando de esta manera los requisitos de multicolinealidad y homocedasticidad necesaria en un AFE. De acuerdo a lo anterior y en función de la revisión de la matriz de determinantes, como se aprecia en la Tabla 2, los ítems que hacen parte de la escala de funcionamiento 
general tienen una correlación directamente proporcional y de magnitud significativa, situación similar a la observada en la escala de funcionamiento emocional (FE); en la escala de síntomas la correlación es significativa pero de magnitud moderada entre los ítems que la componen, pero presentando la linealidad de las correlaciones que es necesaria para poder realizar un análisis factorial.

La rotación por componentes principales se realizó con auto valores superiores a 1 de acuerdo con la regla de Kaiser Myer ${ }^{(6)}$, lo que mostró que el primer componente está conformado por los ítems FG 1: Actualmente, ¿Tiene alguna dificultad para dar un paseo corto fuera de casa? $(\lambda=, 890), F G 2$ : Actualmente, $i$ Tiene que permanecer en la cama o sentado/a en una silla durante el día? $(\lambda=, 866)$ y FG 3 Actualmente, ¿Necesita ayuda para comer, vestirse, bañarse o ir al baño? $(\lambda=, 892)$; que corresponden a la escala de funcionamiento global, y que explican un $56,228 \%$ de la varianza del modelo.

El segundo componente está conformado por ítems que hacen parte de la escala sintomática, que invitan al usuario a responder pensando en su apreciación de síntomas "Durante la semana pasada", en orden de aporte a este factor están los ítems ES 12: ¿Interfirió algún dolor en sus actividades diarias? $(\lambda=, 811)$, ES 5: ¿Ha tenido dolor? $(\lambda=, 7,98)$, ES 10: ¿Ha estado estreñido/a? $(\lambda=, 739)$, ES 9 ¿Ha tenido náuseas? $(\lambda=, 676)$, ES 6 ¿Ha tenido dificultades para dormir? $(\lambda=, 621)$ ES 8: ¿Le ha faltado el apetito? $(\lambda=, 605)$. Finalmente se aprecia que el ítem ES 4: ¿Tuvo asfixia? Hace parte de este componente, pero su aporte es bajo $(\lambda=, 539)$ Este segundo factor explica un $10,582 \%$ de la varianza adicional del modelo e incluye predominantemente ítems de la escala sintomática

En el tercer componente también se indaga sobre la autoevaluación que hace el usuario de su experiencia durante la última semana con respecto a los ítems: ES 11: ¿Estuvo cansado/a? $(\lambda=, 876)$, ES 7: ¿Se ha sentido débil? $(\lambda=, 841)$ FE13. Durante la semana pasada, ¿Se sintió nervioso? $(\lambda=, 748), \mathrm{FE} 14$ : durante la semana pasada ¿Se sintió deprimido/a? ( $\lambda=, 631)$ que conforman la escala de funcionamiento emocional, más dos ítems de a escala sintomática, es importante mencionar que estos dos ítems además de ser síntomas físicos experimentados por los usuarios también hacen parte del reporte sintomatológico que hacen sobre su autoevaluación emocional. Este último factor aporta una explicación adicional del 7,899\%, con lo que puede concluirse que la solución factorial modelada, permite explicar un $74.7 \%$ de la varianza total.

Es importante aclarar que estos dos ítems (ES 7 y ES 11) también presentaban valores Lambda en los otros dos componentes pero su carga factorial fue siempre $\lambda<, 500$ por lo que se puede concluir que estos ítems tienen oportunidades de mejora en cuanto su sensibilidad en la medida pero que funcionan mejor en el tercer componente; una situación similar ocurrió con el ítem ES 4, que presentó una carga factorial media $(\lambda=, 509)$ con el primer componente y bajo con el tercero $(\lambda=, 131)$ situación que pone en evidencia la comunalidad de este ítem con los factores, pero que está influido por un alto error de medida.

Luego de la rotación factorial, se estimaron coeficientes de consistencia interna para la totalidad de la escala, demostrando tanto con un coeficiente Omega, $917^{(15)}$, como con el Alpha de Cronbach ,877(16)

Finalmente, se procedió a considerar los ítems 1 a 14 para la validación de su ajuste al modelo de Rasch. Al respecto, se pudo establecer que, en cuanto al total de la escala, existe un adecuado ajuste para los ítems ya que sus valores Infit: (IMNSQ 
0,99, ZSDT: $-0,3$ ) y Outfit (OMNSQ 1,02, ZSDT-0,2) se encuentran dentro de los rangos establecidos como parámetros de ajuste ${ }^{(18)}$.

Una revisión más específica para cada uno de los ítems puede apreciarse en la tabla 3, en la que se puede observar un índice de correlación biserial puntual, aceptable para la mayoría de los estos, de acuerdo con los lineamientos definidos para él análisis de Rasch ${ }^{(19)}$. Especialmente, se puede apreciar que el ítem 13, se encuentra desajustado ya que presenta tanto desajuste próximo como lejano mostrando valores fuera del rango $(0,8-1,3)^{(18)}$, el análisis de las alternativas de respuesta, muestra que la redacción del ítem y la naturaleza de la calidad de vida del paciente, hacen que la opción de respuesta para nada (puntaje: 0 ) sea escogida como opción de respuesta por la mayoría de personas $(42 \%)$, en contraste la opción de respuesta "todo el tiempo" tiene una tasa de elección de 6\%. Una situación similar ocurre con el ítem 7 "Durante la semana pasada ¿se ha sentido débil?" en la que cerca del 59\% de los participantes marcan las opciones Todo el tiempo y Casi todo el tiempo. Finalmente, el ítem 11 presenta desajuste.

Tabla 3. Coeficientes de correlación de Spearman entre las-escalas y total. 15-PAL

\begin{tabular}{|c|c|c|c|c|c|}
\hline \multirow{2}{*}{ Ítem } & \multicolumn{2}{|c|}{ Infit } & \multicolumn{2}{|c|}{ Outfit } & \multirow[t]{2}{*}{ Rabís } \\
\hline & MSNQ & ZSTD & MSNQ & ZSTD & \\
\hline $\begin{array}{l}\text { Actualmente: } 1 . \text { ¿Tienes alguna dificultad para dar un } \\
\text { paseo corto fuera de casa? }\end{array}$ & 84 & $-1,5$ & ,76 & $.2,0$ &, $75^{* *}$ \\
\hline $\begin{array}{l}\text { Actualmente: } 2 \text {. ¿Tiene que permanecer en la cama o } \\
\text { sentado/a en una silla durante el día? }\end{array}$ & 80 & $-2,0$ & 073 & $-1,4$ &, $77 * *$ \\
\hline $\begin{array}{l}\text { Actualmente: } 3 \text {. ¿Necesita ayuda para comer, vestirse, } \\
\text { bañarse o ir al baño? }\end{array}$ & ,99 &,- 10 & ,97 &,- 20 &, $73 *$ \\
\hline Durante la semana pasada 4. ¿Tuvo Asfixia? & 1,28 & 2,0 & 1.31 & 1,2 &, $55^{*}$ \\
\hline Durante la semana pasada 5. ¿Ha tenido dolor? & 1,07 & 6,0 & 1,07 &, 70 &, $65^{*}$ \\
\hline $\begin{array}{l}\text { Durante la semana pasada } 6 . \text { ¿Ha sentido dificultades } \\
\text { para dormir? }\end{array}$ & 1,01 &, 10 & 1,09 & 80 &, $62 *$ \\
\hline Durante la semana pasada 7 . ¿Se ha sentido débil? &, 50 & $-5,8$ &, 53 & $-4,9$ &, $81 * *$ \\
\hline Durante la semana pasada $8 . ¿$ Le ha faltado el apetito? & ,95 &, 5 & ,94 &,- 5 &, $70^{*}$ \\
\hline Durante la semana pasada 9. ¿Ha tenido nauseas? & 1,31 & 1,6 & 1,3 & 1,1 &, $55^{*}$ \\
\hline Durante la semana pasada 10. ¿Ha estado estreñido/a? & 1,23 & 1,9 & 1,21 & 1,6 &, $56^{*}$ \\
\hline Durante la semana pasada 11. ¿Estuvo Cansado? & ,58 & $-4,7$ & ,56 & -4.5 &, $82 * *$ \\
\hline $\begin{array}{l}\text { Durante la semana pasada } 12 \text {. ¿Ha sentido dificultades } \\
\text { para dormir? }\end{array}$ & 1,03 & ,30 & ,99 &, 1 &, $72 *$ \\
\hline Durante la semana pasada 13. ¿Se sintió nervioso/a? & 1,23 & 3,4 & 1,07 & 4,6 &, $48^{*}$ \\
\hline Durante la semana pasada 14. ¿Se sintió deprimido/a? & ,78 & $-2,1$ & ,85 & $-1,3$ &, $69^{*}$ \\
\hline
\end{tabular}

** La correlación es significativa en un IC del 99\%

* La correlación es significativa en un IC del 95\%

Por lo que sería ideal hacer un ajuste en la redacción de los ítems para que estos discriminen de manera precisa el rasgo que pretenden medir. 


\section{Discusión}

La validación del EORTC QLQ-C15-PAL para Colombia demostró propiedades psicométricas satisfactorias al aplicarse en pacientes con enfermedad oncológica avanzada; estos hallazgos son similares a los encontrados en un estudio realizado en Chile ${ }^{(20)}$. La correlación inter-escalas demuestra que el EORT QLQ 15 PAL presenta consistencia entre las escalas que lo componen. Los hallazgos de este estudio en los que los ítems de la escala de síntomas no se ajustan al factor, sino que se suman a los ítems de la escala de funcionamiento emocional, son coherentes con los hallazgos en muestras españolas de pacientes con cáncer óseo en los que la correlación ítemítem; ítem-escala y los coeficientes Alfa de Cronbach mostraron baja consistencia interna $^{(21)}$. Estos mismos autores encontraron mediante validez convergente, que los ítems 11 y 7 no tenían una adecuada discriminación al presentarse juntos en una misma prueba en español; tal y como ocurre con nuestros datos, ya que la interpretación que se hace de su sintaxis en población latinoamericana hace que las palabras se interpreten como sinónimos de una misma experiencia (la percepción de falta de energía).

En el estudio realizado en pacientes mexicanos ${ }^{(10)}$, con una muestra heterogénea de 83 pacientes con confirmación histológica de diferentes tipos de cáncer, los autores reportaron una buena validez convergente y discriminante, el coeficiente de correlación entre los ítems dentro de la misma escala osciló entre 0,51 y 0,65 para la escala de síntomas y 0,58 y 0,78 para la escala funcional, en contraste con nuestros hallazgos que muestran una mejor consistencia interna. El alfa de Cronbach varió entre 0,67 y 0,88 , concluyendo que el QLQ C15 PAL es una herramienta confiable y válida para la medición de la calidad de vida en pacientes mexicanos con cáncer terminal ${ }^{(10)}$.

Por otro lado, versiones en idiomas diferentes al español han llegado a conclusiones similares, en cuanto a nuestros hallazgos; por ejemplo, para población turca ${ }^{(23)}$ la consistencia interna, fue superior a la nuestra. Sin embargo, ellos han calculado un coeficiente Alpha para cada dimensión, aun cuando dos de ellas cuentan con pocos ítems por lo que podrían presentar sesgo. Estos mismos investigadores, demostraron una correlación ítem-escala similar a la nuestra, presentando también baja precisión para los ítems de la escala sintomática ${ }^{(22)}$. Si bien no se ha usado la técnica de AFE para las pruebas, la versión Árabe validada en población jordana, muestra una aproximación a la agrupación de nuestros resultados, mostrando una alta consistencia en los ítems de la escala de funcionalidad Global; también una consistencia en los síntomas fatiga, dolor y otros síntomas físicos; es consistente que la discriminación más baja esté en los ítems 4,5 y 7 que se refieren al dolor, la sensación de asfixia y debilidad ${ }^{(23)}$, lo que también sugiere que es posible mejorar la especificidad de la versión original del EORT QLQ 15 PAL, para reinterpretar de manera operacional estos síntomas, ya que la evidencia nos muestra que son ítems fundamentales para la medida, dada su correlación con los puntajes totales; pero quizás no son descriptores precisos de la experiencia de las personas que requieren atención paliativa. 


\section{Conclusiones}

La validación del EORTC QLQ-C15-PAL demostró propiedades psicométricas satisfactorias al aplicarse en pacientes con enfermedad oncológica avanzada en Colombia. La correlación inter-escalas demuestra que el EORT QLQ 15 PAL presenta consistencia entre las escalas que lo componen.

Se plantea para estudios posteriores realizar ajuste en la redacción de los ítems 7 , 11 y 13 para que estos discriminen de manera precisa el rasgo que pretenden medir, usando descriptores claros y con los que los usuarios se muestren más familiarizados o incluso estudiar la posibilidad de considerar solo uno de los ítems referentes al agotamiento/falta de energía/cansancio percibido.

\section{Referencias bibliográficas}

1. Bray F, Ferlay J, Soerjomataram I, Siegel RL, Torre LA, Jemal A. Global cancer statistics 2018: GLOBOCAN estimates of incidence and mortality worldwide for 36 cancers in 185 countries. CA Cancer J Clin 2018;68:394-424. https://doi.org/10.3322/caac.21492

2. Cuidados paliativos [Internet]. OMS. 2020 [citado 16 octubre 2020]. Disponible en: https://www.who.int/es/news-room/fact-sheets/detail/palliative-care\#: :text=Los\%20 cuidados $\% 20$ paliativos $\% 20$ constituyen $\% 20$ un, a $\% 20$ una $\% 20$ enfermedad $\% 20$ potencialmente $\% 20$ mortal.

3. Aaronson NK, Ahmedzai S, Bergman B, Bullinger M, Cull A, Duez NJ, et al. The European Organization for Research and Treatment of Cancer QLQ-C30: A qualityof-life instrument for use in international clinical trials in oncology. J Natl Cancer Inst [Internet]. 1993 Mar 3;85:365-76. https://doi.org/10.1093/jnci/85.5.365

4. Sousa VD, Rojjanasrirat W. Translation, adaptation and validation of instruments or scales for use in cross-cultural health care research: a clear and user-friendly guideline. J Eval Clin Pract 2011;17:268-74. https://doi.org/10.1111/j.1365-2753.2010.01434.x

5. Coulacoglou, C., \& Sklofske D. Psychometrics and psychological assessment.1st Edition. Londres: Academic Press; 2017.

6. Rust J, Susan G. Modern psychometrics the science of psychological assessment. Londres: Psychology Press, 2014.

7. Keith C. An Introduction to psychological assesssment and psychometrics. Second. Londres: Sage, 2010.

8. Hughes DJ, Booth T IP. The Wiley Handbook of Psychometric Testing: A multidisciplinary reference on survey, scale and test development. Nueva YorK: Wiley-Blackwell; 2018. 2018.

9. Coolican H. Métodos de investigación y estadística en psicología. 3 ed. México D.F.: Manual Moderno; 2005.

10. Suárez-Del-Real Y, Allende-Pérez S, Alférez-Mancera A, Rodríguez RB, Jiménez-Toxtle S, Mohar A, et al. Validation of the Mexican-Spanish version of the EORTC QLQC15-PAL questionnaire for the evaluation of health-related quality of life in patients on palliative care. Psychooncology 2011;20:889-96. https://doi.org/10.1002/pon.1801

11. Sánchez R, Venegas M, Otero J, Sánchez O. Adaptación transcultural de dos escalas para medir la calidad de vida en pacientes con cáncer en Colombia: EORTC QLQ-C30 y QLQ-BR23. Rev Colomb Cancerol [Internet]. 1969;13(4 SE-Artículos de investigación/ originales):205-12. 
12. Caissie A, Culleton S, Nguyen J, Zhang L, Zeng L, Holden L, et al. EORTC QLQC15-PAL quality of life scores in patients with advanced cancer referred for palliative radiotherapy. Support Care Cancer 2012;20:841-8. https://doi.org/10.1007/s00520-0111160-6

13. Karnofsky D a, Abelmann WH, Craver LF, Burchenal JH. The use of the nitrogen mustards in the palliative treatment of carcinoma. Cancer 1948;1:634-56. https://doi. org/10.1002/1097-0142(194811)1:4<634::AID-CNCR2820010410>3.0.CO;2-L

14. Péus D, Newcomb N, Hofer S. Appraisal of the Karnofsky Performance Status and proposal of a simple algorithmic system for its evaluation. BMC Med Inform Decis Mak 2013;13. https://doi.org/10.1186/1472-6947-13-72

15. Ventura-León, José Luis, Caycho-Rodríguez, Tomás, El coeficiente Omega: un método alternativo para la estimación de la confiabilidad. Revista Latinoamericana de Ciencias Sociales, Niñez y Juventud 2017;15:625-7. [Acceso 1 de abril de 2021] Disponible en: : https://www.redalyc.org/articulo.oa?id=77349627039

16. Cronbach LJ. Coefficient alpha and the internal structure of tests. Psychometrika 1951;16:297-334. https://doi.org/10.1007/BF02310555

17. Jose Muñiz. Las teorías de los test: teoria clasica y teoria de respuesta a los items. Papeles de Psicólogo 2010;31:57-66.

18. tabla 4Linacre J. Rasch power analysis: size vs. significance: infit and outfit mean-square and standardized chi-square fit statistic. Rasch Meas Trans 2003;17:918

19. Linacre J. Rasch Measurement. Transactions of the Rasch Measurement SIG American Educational Research Association. Rasch Meas Trans 2006;20(1).

20. Rojas-Concha L, Hansen MB, Petersen MA, Groenvold M. Erratum: Content validation of the EORTC QLQ-C15-PAL with advanced cancer patients and health care professionals from palliative care services in Chile. BMC Palliat Care 2020;19:1-10. https://doi. org/10.1186/s12904-020-00586-1

21. Arraras JI, De La Vega FA, Asin G, Rico M, Zarandona U, Eito C, et al. The EORTC QLQ-C15-PAL questionnaire: Validation study for Spanish bone metastases patients. Qual Life Res 2014;23:849-55. https://doi.org/10.1007/s11136-013-0511-9

22. Ozcelik H, Guzel Y, Sonmez E, Aksoy F, Uslu R. Reliability and validity of the Turkish version of the EORTC QLQ-C15-PAL for patients with advanced cancer. Palliat Support Care 2016;14:628-34. https://doi.org/10.1017/S1478951516000195

23. Alawneh A, Yasin H, Khirfan G, Qayas BA, Ammar K, Rimawi D, et al. Psychometric properties of the Arabic version of EORTC QLQ-C15-PAL among cancer patients in Jordan. Support Care Cancer 2016;24:2455-62. http://dx.doi.org/10.1007/s00520-0153018-9 